ОБОСНОВАНИЕ. Ввиду неуклонного роста заболеваемости патологиями щитовидной, околощитовидных желез и других органов шеи, радикальное лечение которых может привести к развитию гипопаратиреоза, прогнозируется значимое увеличение числа больных с данным заболеванием. Оценка эпидемиологических и клинических особенностей хронического гипопаратиреоза необходима для прогнозирования социально значимых осложнений и повышения качества оказания медицинской помощи.

ЦЕЛЬ. Оценить клинико-демографические характеристики и эффективность использования различных схем медикаментозной терапии у пациентов с хроническим послеоперационным и нехирургическим гипопаратиреозом с помощью базы данных НМИЦ эндокринологии.

МЕтОДЫ. Проведено одномоментное обсервационное сплошное исследование с использованием базы данных пациентов с хроническим послеоперационным и нехирургическим гипопаратиреозом, в которое включены 293 пациента из 61 региона РФ. Анализ данных проведен в июне 2020 г. Количественные признаки представлены медианами (Ме) и квартилями (Q1; Q3); описательная статистика качественных признаков - абсолютными и относительными частотами.

РЕзУЛЬтАТЫ. По результатам анализа базы данных, чаще всего гипопаратиреоз зафиксирован уженщин (85\%) в возрасте 43 [32; 52] лет после перенесенного хирургического вмешательства по поводу патологии щитовидной железы. Менее половины обследованных пациентов имеют целевые показатели кальция и фосфора сыворотки крови (31 и 47\% соответственно), несмотря на проводимое лечение. Полноценное инструментальное обследование, позволяющее составить полную картину наличия осложнений, проведено в 58\% случаев (n=169). Нарушения со стороны почек выявлены у 38\%, органов зрения - у 14\%, кальцификация головного мозга - у 10\%, изменения сердечного ритма - у 4\% и психоневрологического статуса - у 6\% пациентов. Стандартную терапию (комбинацию активных метаболитов/аналогов витамина D и препаратов кальция) принимали 75\% пациентов.

ЗАКЛЮчЕНИЕ. Анализ крупных баз данных пациентов с хроническим гипопаратиреозом - необходимый инструмент для определения прогностических маркеров, в том числе с целью профилактики развития инвалидизирующих осложнений и формирования критериев компенсации заболевания.

КЛЮЧЕВЫЕ СЛОВА: гипопаратиреоз; гипокальчиемия; терапия; кальций; витамин D; тиреоиээктомия.

\title{
EVALUATION OF CHRONIC HYPOPARATHYROIDISM COURSE ACCORDING TO THE DATABASE OF ENDOCRINOLOGY RESEARCH CENTRE
}

\author{
(c) Elena V. Kovaleva*, Alina R. Ajnetdinova, Anna K. Eremkina, Natalia G. Mokrysheva
}

Endocrinology Research Centre, Moscow, Russia

BACKGRAUND: Currently high frequency of thyroid or parathyroid surgery is associated with significant increased incidence of hypoparathyroidism. Evaluation of the epidemiological and clinical features of chronic hypoparathyroidism is necessary to predict social-significant complications and to improve the quality of medical care.

AIMS: To estimate clinical and demographic features, different regimens and efficacy of conventional therapy in patients with chronic postsurgical and nonsurgical hypoparathyroidism.

MATERIALS AND METHODS: The cross-sectional, observational, continuous study was carried out based on Database of patients with chronic postsurgical and nonsurgical hypoparathyroidism of Endocrinology Research Centre, Moscow. 293 patients from 61 regions of the Russian Federation were included in this study. Statistical analysis was done on June 2020. The descriptive statistics are presented by medians (Me) and the first and third quartiles (Q1; Q3) and by absolute and relative frequencies.

RESULTS: Hypoparathyroidism was most often recorded in women (85\%) at the age of 43 [32; 52] years, after thyroid surgery. Less than a half of the study group had target indicators of serum calcium and phosphate levels $(31 \%$ and $47 \%$, respectively) despite ongoing treatment. A complex instrumental examination for the disease complications was performed in $58 \%$ of cases $(n=169)$. Among them, kidney disorders were detected in $38 \%$, visual disturbance in $14 \%$, brain calcification in $10 \%$, 
arrhythmias in $4 \%$ and neuropsychiatric symptoms in $6 \%$. Conventional therapy with calcium supplements and activated vitamin $\mathrm{D}$ analogues was noted in $75 \%$ of patients.

CONCLUSIONS: The analysis of large databases of patients with chronic hypoparathyroidism is a necessary tool for determining of optimal clinical and therapeutic approaches, as well as prognostic markers of disease complications.

KEYWORDS: hypoparathyroidism; hypocalcaemia; therapy; calcium; vitamin D; thyroidectomy.

\section{ОБОСНОВАНИЕ}

Гипопаратиреоз - эндокринное заболевание, характеризующееся сниженной продукцией паратиреоидного гормона (ПТГ) околощитовидными железами (ОЩЖ), что сопровождается нарушениями фосфорно-кальциевого обмена. В последнее время изучению различных аспектов данной патологии уделяется много внимания. В литературе представлены результаты анализа крупных национальных баз данных США, Дании, Норвегии и Италии, в соответствии с которыми распространенность гипопаратиреоза составляет в среднем 23-46 случаев на 100 тыс. населения с преобладанием послеоперационного гипопаратиреоза (63-91\%). Сведения по распространенности гипопаратиреоза на территории Российской Федерации отсутствуют.

Ввиду неуклонного роста заболеваемости раком щитовидной железы, первичным гиперпаратиреозом и другими патологиями органов шеи, радикальное лечение которых может привести к развитию гипопаратиреоза, прогнозируется неуклонный рост числа больных с этой патологией. Важно, что чаще раком щитовидной железы страдают лица трудоспособного возраста.

Развитие хронического гипопаратиреоза любой этиологии требует пожизненного применения многокомпонентной терапии, а также тщательного мониторинга и индивидуального подхода к выбору оптимальной тактики. В отсутствие адекватного динамического наблюдения значимо возрастают риски осложнений, в частности нефрокальциноза, нефролитиаза с развитием почечной недостаточности, кальцификации мягких тканей и головного мозга. «Неконтролируемый» гипопаратиреоз приводит к сердечно-сосудистым осложнениям, зрительным нарушениям, патологии костно-мышечной системы, развитию нейрокогнитивных расстройств и резкому снижению качества жизни пациентов.

\section{ЦЕЛЬ}

Целью настоящего исследования являлась оценка клинико-демографических характеристик, частоты развития осложнений и эффективности использования различных схем медикаментозной терапии у пациентов с хроническим послеоперационным и нехирургическим гипопаратиреозом с помощью базы данных НМИЦ эндокринологии.

\section{МЕТОДЫ}

Дизайн исследования

Проведено одномоментное обсервационное сплошное исследование с использованием базы данных пациентов с хроническим послеоперационным и нехирургическим гипопаратиреозом отделения патологии околощитовидных желез ФГБУ «НМИЦ эндокринологии» Минздрава России.

\section{Критерии соответствия}

В исследование вошли пациенты с хроническим послеоперационным и нехирургическим гипопаратиреозом любой этиологии, исключая патологию, описанную в критериях исключения, без ограничений по возрасту и полу. Диагноз хронического гипопаратиреоза устанавливался на основании результатов лабораторного исследования: стойкая гипокальциемия (уровень кальция сыворотки крови ниже нижней границы референсного диапазона) в сочетании со снижением уровня паратиреоидного гормона (ПТГ) ниже референсного диапазона в течение более чем 6 месяцев наблюдения. К критериям исключения отнесены: функциональный гипопаратиреоз (в результате нарушения обмена магния); транзиторный гипопаратиреоз (длительность заболевания менее 6 мес). Диагноз идиопатического гипопаратиреоза устанавливался на основании наличия гипокальциемии в сочетании со снижением уровня ПТГ при невозможности подтверждения этиологии заболевания (отсутствие результатов генетического исследования).

\section{Условия проведения}

Объектом исследования являлась база данных НМИЦ эндокринологии (стационарные и амбулаторные эпизоды) за период 2016-2020 гг. Анализировались амбулаторные и стационарные истории болезни с кодами МКБ-10: E20.0, E20.8, E20.9, E89.2. Все клинические, лабораторные и инструментальные манипуляции, выполненные в рамках данной работы, были проведены на базе нескольких учреждений (в том числе по месту жительства пациента). Для всех лабораторных показателей использовался референсный интервал (РИ) местной лаборатории с обязательным указанием нижней и верхней границ. Наличие или отсутствие осложнений хронического гипопаратиреоза регистрировалось путем обработки результатов лабораторно-инструментального обследования. Каких-либо специфических факторов, способных повлиять на внешнюю обобщенность выводов исследования, не выявлено.

Согласно дизайну исследования, проведен анализ следующих показателей: пол, возраст на момент постановки диагноза, длительность заболевания и его этиология (в том числе патология органов шеи для послеоперационного гипопаратиреоза); результаты лабораторных методов обследования: биохимического исследования крови (уровень кальция общего $\left(\mathrm{Ca}_{\text {общ.), }}\right)$ альбумин скорректированного ( $\left(\mathrm{a}_{\text {скорр. }}\right)$ и ионизированного $\left(\mathrm{Ca}^{++}\right)$, фосфора, креатинина с расчетом СКФ по ЕРІ, 
магния, креатинфосфокиназы (КФК)) и биохимического исследования мочи (суточная кальциурия). Анализировались также результаты инструментальных методов: ультразвуковое исследование (УЗИ) почек; компьютерная томография головного мозга; заключения специалистов (офтальмолога, психоневролога, кардиолога); используемые схемы лечения. На основании результатов лабораторно-инструментального обследования и заключения специалистов вносилась информация об осложнениях хронического гипопаратиреоза со стороны почек, сердечно-сосудистой, нервной систем, органов зрения.

Продолжительность исследования

Анализ данных проведен в июне 2020 г.

\section{Описание медицинского вмешательства}

Специалисты работали с имеющимися результатами лабораторно-инструментального обследования, выполненного пациентом амбулаторно или во время госпитализации в специализированном стационаре.

\section{Этическая экспертиза}

Локальным этическим комитетом ФГБУ «Национальный медицинский исследовательский центр эндокринологии» Минздрава России, согласно протоколу №18 заседания Комитета от 11.10.2017 г., постановлено, что планируемая научная работа соответствует этическим стандартам добросовестной клинической практики и может быть проведена на базе отделения патологии околощитовидных желез ФГБУ «НМИЦ эндокринологии» Минздрава России. Пациенты подписывали информированное согласие на добровольное участие в исследовании и обработку личных данных.

Принципы расчета размера выборки: расчет размера выборки не требовался.

Статистический анализ проводился в программных пакетах Statistica 13.0 (StatSoft, США) и SPSS (IBM, США). Описательная статистика количественных признаков представлена медианами и квартилями (в формате Me [Q1; Q3]), описательная статистика доз лекарственных препаратов представлена в виде среднего, минимального и максимального значений, описательная статистика качественных признаков абсолютными и относительными частотами. Для анализа соответствия распределений количественных признаков нормальному закону применялся критерий Шапиро-Уилка. Для сравнения двух независимых групп по количественным признакам использовали критерий Манна-Уитни (U-test). Критический уровень значимости при проверке статистических гипотез принимался равным 0,05. При множественных сравнениях применялась поправка Бонферрони путем коррекции критического уровня значимости. Доверительные интервалы (ДИ) для частот рассчитывались методом Клоппера-Пирсона.

\section{РЕЗУЛЬТАТЫ}

Объекты (участники) исследования

В исследование включены 293 пациента с верифицированным хроническим гипопаратиреозом раз- личной этиологии (250 женщин, 43 мужчины). Возраст больных на момент постановки диагноза составил 48 лет [36; 63], женщин - 49 лет [38; 64], мужчин 34 года $[17 ; 53]$.

С учетом поправки Бонферрони пациенты с послеоперационным гипопаратиреозом $\left(P_{0}=0,017\right)$ имели более поздний возраст манифестации заболевания по сравнению с нехирургическим гипопаратиреозом (50 vs 23 года, $\mathrm{p}<0,001, \mathrm{U}$-test). Ме возраста на момент постановки диагноза для группы послеоперационного гипопаратиреоза составила 43 года [32; 52], в данной группе преобладали женщины - до 91\%, 95\% ДИ 88-95\% (228). В группе нехирургического гипопаратиреоза Ме возраста на момент постановки диагноза была меньше - 17 лет [6; 31]. Различий по полу в данной группе не обнаружено 1:1 (22/23).

При оценке распределения манифестации заболевания по возрастам частота выявления гипопаратиреоза среди лиц моложе 18 лет составила 12,3\% (95\% дИ 8,8-16,6\%), от 18 до 34 лет - 25,6\% (95\% ДИ 20,7-31), от 35 до 44 лет - 23,2\% $(95 \%$ ДИ 18,5-28,5) и от 45 до 54 лет - 20,1\% (95\% ДИ 15,7-25,2), постепенное снижение отмечается после 54 лет - до 14\% (95\% ДИ 10,2-18,5) и до 4,1\% (95\% ДИ 2,1-7) у лиц старше 65 лет (рис. 1). У двух пациентов четкая информация о начале заболевания отсутствует. Среди пациентов с возрастом манифестации заболевания младше 18 лет было 13 больных с послеоперационным гипопаратиреозом и 16 - с нехирургическими формами заболевания.

Ме длительности заболевания для послеоперационного гипопаратиреоза - 6 лет $[4 ; 11,8]$, для нехирургического гипопаратиреоза - 4 года $[2 ; 8,5]$.

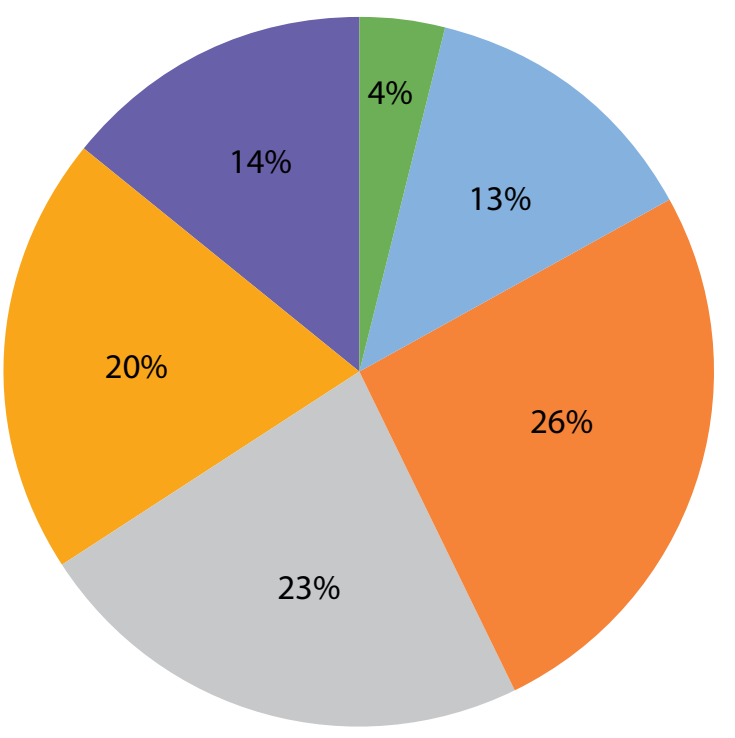

$<18$ лет

18-34 года

35-44 года

45-54 года

55-64 года

более 65 лет

Рисунок 1. Распределение пациентов с гипопаратиреозом по возрастам. 


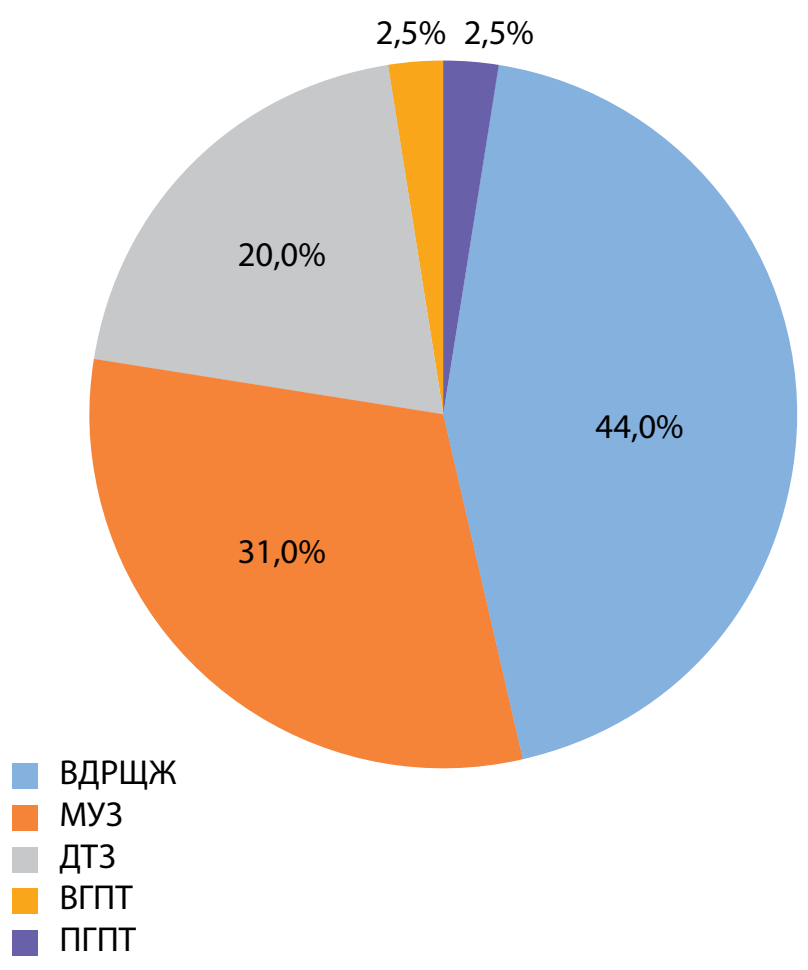

Рисунок 2. Спектр заболеваний, хирургическое лечение которых сопровождалось развитием хронического послеоперационного гипопаратиреоза.

\section{Этиология}

Самой частой причиной развития заболевания являлось повреждение и/или удаление ОЩЖ в ходе хирургического вмешательства на органах шеи с развитием послеоперационного гипопаратиреоза (85\%, 95\% ДИ 80-89). Среди других более редких причин - аутоиммунное повреждение ткани ОЩЖ в рамках аутоиммунного полигландулярного синдрома (АПС) 1 типа с подтвержденной мутацией в гене AIRE у 8 пациентов (3\%, 95\% ДИ 1,2-5,3), аутосомно-доминантная гипокальциемия у 5 (2\%, 95\% ДИ 80-89), гипопаратиреоз в составе многокомпонентных наследственных синдромов у 2 (1 пациент с синдромом ДиДжорджи и 1 пациент с синдромом Кенни-Каффи). У 30 пациентов (10\%, 95\% ДИ 7-14,3) гипопаратиреоз был расценен как идиопатический.

В нашем исследовании не было ни одного случая повреждения ткани ОЩЖ вследствие лучевой нагрузки, а также в результате инфильтративного или метастатического заболевания.

Причины развития послеоперационного хронического гипопаратиреоза

В большинстве случаев к послеоперационному гипопаратиреозу приводили хирургические вмешательства по поводу высокодифференцированного рака щитовидной железы (ВДРщЖ) - 44\% (95\% ДИ 37-50), многоузлового зоба (МУЗ) - в 31\% (95\% ДИ 26-38), диффузного токсического зоба (ДТЗ) - в 20\% случаев (95\% ДИ 15-25). Операции проводились в объеме тиреоидэктомии (у 235 пациентов), в том числе с центральной и/или боковой лимфодиссекцией (у 45 пациентов). У 6 больных выполнена тотальная паратиреоидэктомия (ПТЭ) по поводу вторичного гиперпаратиреоза (ВГПТ) вследствие диализной стадии хронической болезни почек (ХБП5Д), среди них 1 пациенту проведена аутотрансплантация

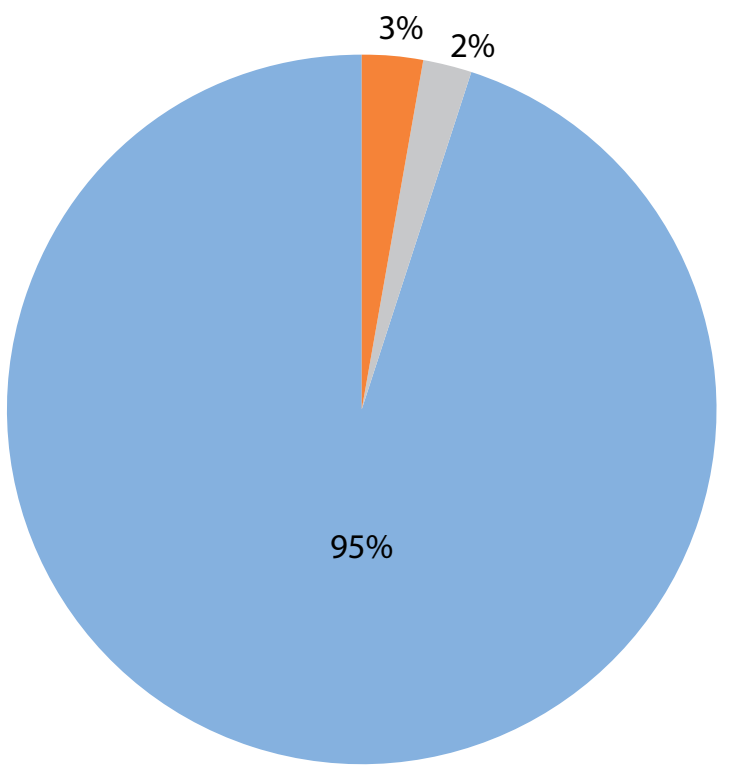

Тиреоидэктомия

Тотальная ПТЭ

Сочетанные вмешательства (селективная ПТЭ и геми/тиреоидэктомия)

Рисунок 3. Частота развития гипопаратиреоза в зависимости от объема хирургического лечения.

ткани ОЩЖ в мышцу предплечья без удовлетворительной функции трансплантата. У 6 пациентов гипопаратиреоз развился после сочетанных операций: у 4 после ПТЭ по поводу первичного гиперпаратиреоза (ПГПТ) совместно с гемитиреоидэктомией по поводу одноузлового зоба и у 2 пациентов - после ПТЭ по поводу ПГПТ совместно с тиреоидэктомией по поводу МУз (рис. 2, 3). В 12\% (95\% ДИ 8-17) послеоперационный гипопаратиреоз развился в результате повторного вмешательства на органах шеи.

\section{Данные лабораторных методов исследования}

У всех пациентов с гипопаратиреозом были доступны значения Са общ. Ме 2,09 ммоль/л $[1,87 ; 2,25]$, уро-

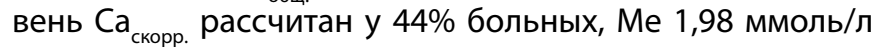
$[1,79 ; 2,17]$. Показатели уровней фосфора, магния и суточной кальциурии были доступны в 80, 48 и 48\% соответственно. Уровни Са общ. находились в пределах целевого диапазона (2,1-2,3 ммоль/л) лишь в $31 \%$ случаев (95\% ДИ 25-37), тогда как у половины пациентов наблюдалась гипокальциемия. Гиперкальциемия зафиксирована в 19\% (95\% ДИ 14-24). Уровни фосфора сыворотки крови в пределах целевого диапазона определялись у 47\% пациентов (95\% ДИ 40-54), при этом значимая гиперфосфатемия (повышение уровня фосфора сыворотки крови свыше 2,0 ммоль/л) отмечена у 12\% (95\% ДИ 8-17). В большинстве случаев (75\%, 95\% ДИ 67-82) показатели магния сохранялись в референсном диапазоне. Повышение суточной экскреции кальция в обследованной группе >8 ммоль/сут выявлено в 26\% наблюдений (95\% ДИ 19-35), а гипокальциурия - в $24 \%$ (95\% ДИ 17-32). Результаты анализа основных показателей фосфорно-кальциевого обмена у пациентов с хроническим гипопаратиреозом отражены в таблице 1. 
Таблица 1. Лабораторные показатели у пациентов с гипопаратиреозом.

\begin{tabular}{|c|c|c|c|}
\hline $\begin{array}{c}\text { Лабораторные } \\
\text { показатели }\end{array}$ & Me [Q1; Q3] & Целевой диапазон & $\begin{array}{c}\text { Количество пациентов } \\
\text { (частота, \%) }\end{array}$ \\
\hline \multirow{3}{*}{$\begin{array}{l}\text { Кальций общий, ммоль/л } \\
293 \text { (100\%) }\end{array}$} & $1,88[1,77 ; 1,98]$ & $<2,1$ & $145(49,7)$ \\
\hline & $2,2[2,13 ; 2,25]$ & $2,1-2,3$ & $88(30,1)$ \\
\hline & $2,41[2,36 ; 2,5]$ & $>2,3$ & $59(20,2)$ \\
\hline \multirow{3}{*}{$\begin{array}{l}\text { Фосфор, ммоль/л } \\
234(80 \%)\end{array}$} & $1,35[1,25 ; 1,43]$ & $0,74-1,52$ & $110(47)$ \\
\hline & $1,69[1,61 ; 1,8]$ & $>1,52$ & $94(40,2)$ \\
\hline & $2,22[2,09 ; 2,62]$ & $>2,0$ & $29(12,4)$ \\
\hline \multirow{2}{*}{$\begin{array}{l}\text { Магний, ммоль/л } \\
139(48 \%)\end{array}$} & $0,65[0,63 ; 0,67]$ & $<0,7$ & $35(25,2)$ \\
\hline & $0,78[0,74 ; 0,82]$ & $0,7-1,05$ & $104(74,8)$ \\
\hline \multirow{3}{*}{$\begin{array}{l}\text { Кальций в суточной моче, } \\
\text { ммоль/сут } \\
140(48 \%)\end{array}$} & $1,4[0,85 ; 1,92]$ & $<2,5$ & $34(24,3)$ \\
\hline & $4,8[3,86 ; 6,37]$ & $2,5-8$ & $69(49,3)$ \\
\hline & $10,6[9,2 ; 13,36]$ & $>8$ & $37(26,4)$ \\
\hline
\end{tabular}

Сучетомпоправки Бонферрони $\left(\mathrm{P}_{0}=0,017\right)$ при сравнении уровня кальциемии в группах с послеоперационным и нехирургическим гипопаратиреозом по концентраци-

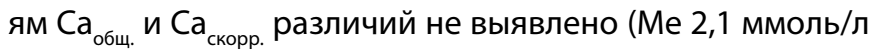
[1,89; 2,24] vs Me 2,015 ммоль/л [1,79; 2,27], $\mathrm{p}=0,402$; Me 1,98 ммоль/л $[1,8 ; 2,17]$ vs 1,95 ммоль/л $[1,75 ; 2,16]$, $\mathrm{p}=0,446$ соответственно, U-test).

Оценка фильтрационной функции почек проведена 179 пациентам (61\%). Среди них снижение СКФ менее 60 мл/мин/м² выявленоу 38 пациентов (21\%, 95\% ДИ 15-28), ХБП 4 и 5 ст. со снижением СКФ менее 30 мл/мин $/ \mathrm{M}^{2}$ диагностирована у 5 больных (3\%, 95\% ДИ 1-6).

Данные об уровне 25(OH)витамина D представлены у 42\% больных. При этом целевые для общей популяции значения 25(OH)витамина D (более 30 нг/мл) зафиксированы в более чем половине наблюдений (59\%, 95\% ДИ 50-68). У 2 больных на фоне регулярной терапии колекальциферолом уровни витамина D составили более 100 нг/мл.

Уровни КФК как неспецифического маркера миопатии были доступны у 51 пациента (17\%), среди них в 35\% случаев (95\% ДИ 22-50) отмечено повышение показателя выше РИ (>168 ед/л). Сопоставление групп по кальциемии и уровню КФК показало достоверно более высокий уровень КФК среди пациентов с более выраженной гипокальциемией (Me Ca $\mathrm{C}_{\text {скорр. }} 1,78[1,46 ; 2,06]$ vs $2,05[1,82 ; 2,17]$ ммоль/л, $p=0,034, \mathrm{U}$-test).

\section{Осложнения заболевания}

Инструментальное обследование с целью выявления осложнений заболевания проведено 58\% пациентов $(n=169)$, в связи с чем расчет частоты выявления тех или иных осложнений производился по отношению к обследованной группе пациентов (табл. 2).

\section{Схемы и дозы лекарственной терапии}

Стандартную схему лечения в виде комбинации активных метаболитов/аналогов витамина D и препаратов кальция принимали 75\% пациентов (95\% ДИ 70-80). Средние дозы альфакальцидола составили 1,74 мкг/сут (min 0,25; $\max 6)$, кальцитриола - 1,1 мкг/сут (min 0,25; $\max 2,5)$. Нативный витамин D в качестве дополнения к стандартной терапии назначался 69 больным (24\%, 95\% ДИ 19-29) в средней дозе 18374 ME/нед (min 142; max 300 000). Использование схемы «колекальциферол + препараты кальция» в качестве лечения гипопаратиреоза отмечено у 12 больных, при этом средняя доза колекальциферола была 43625 ME/нед (min 7000; max 300 000). «Дигидротахистерол + препараты кальция» принимали 11 пациентов.

Препараты кальция назначались $87 \%$ больным (95\% ДИ 83-91) в среднесуточной дозе 1837 мг/сут (min 250; max 7500), из них 19\% (95\% ДИ 17-27) больных получали свыше 3000 мг/сут. Среди различных солей наиболее часто назначался карбонат кальция (86\%, 95\% ДИ 81-89), 5 пациентов получали цитрат кальция в средней дозе 1300 мг/сут.

Таблица 2. Частота осложнений хронического гипопаратиреоза по результатам анализа базы данных НМИЦ эндокринологии.

\begin{tabular}{lcc}
\hline \multicolumn{1}{c}{ Осложнение } & Количество пациентов, & Относительная частота, \\
\%=169
\end{tabular}


Гидрохлоротиазид для коррекции гиперкальциурии применялся у 22 пациентов в средней дозе 30 мг/сут (min 6,25; $\max 75)$. С целью коррекции гипомагниемии препараты магния были назначены 38 больным (13\%, 95\% ДИ 9-17), в среднем по 440 мг/сут. Использование Севеламера для коррекции гиперфосфатемии отмечено только в двух наблюдениях. Терапию рекомбинантным человеческим ПТГ - ПТГ (1-34) получали 2 пациента в средней дозе 25 мг/сут подкожно.

Проведена оценка влияния различных доз лекарственных препаратов и рисков развития осложнений заболевания. С учетом поправки Бонферрони $\left(\mathrm{P}_{0}=0,013\right)$ установлено, что большие суточные дозы альфакальцидола умеренно коррелируют с увеличением суточной кальциурии ( $p=0,004 ; r=0,260$; метод Спирмена), при этом взаимосвязи между дозами альфакальцидола с показате-

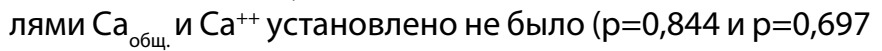
соответственно, метод Спирмена). Было отмечено, что больные, принимающие гидрохлоротиазид, в отличие от пациентов, не принимающих диуретик, использовали большие дозы препаратов кальция (2900 [2000; 3000] vs 1500 [1000; 2000] мг/сут; p<0,001; U-test). Суточные дозы препаратов кальция не были ассоциированы с повышением суточной кальциурии ( $p=0,395 ;$ метод Спирмена).

\section{Нежелательные явления}

В исследовании не планировалось отслеживать нежелательные явления, в том числе оценивать безопасность медикаментозной терапии.

\section{ОБСУЖДЕНИЕ}

\section{Резюме результатов исследования}

По результатам проведенного исследования отмечено, что чаще всего гипопаратиреозом заболевают женщины (85\%, 95\% ДИ 81-89) в возрасте от 30 до 50 лет, после перенесенного хирургического вмешательства на области шеи. По данным биохимического исследования крови только 31\% (95\% ДИ 25-37) пациентов имеют целевые показатели кальция и 47\% (95\% ДИ 40-54) фосфора сыворотки крови.

Обследование в необходимом объеме имеется лишь у $58 \%$ пациентов $(n=169)$. Среди осложнений заболевания в обследуемой группе преобладали нарушения со стороны почек (38\%), реже диагностировались патология органов зрения (14\%), кальцификация головного мозга (10\%), нарушения сердечного ритма (4\%) и психоневрологические нарушения (6\%).

При анализе терапии заболевания получено, что стандартную схему лечения в виде комбинации активных метаболитов/аналогов витамина D и препаратов кальция на момент включения в исследование принимали 75\% (95\% ДИ: 70-80\%) пациентов. Большие суточные дозы альфакальцидола ассоциировались с увеличением показателей суточной кальциурии ( $p=0,004 ; r=0,260 ;$ метод Спирмена). Пациенты, принимающие гидрохлоротиазид, в отличие от больных, не принимающих диуретик, использовали большие дозы препаратов кальция ( $p<0,001$; U-test).

Обсуждение результатов исследования

Полученные данные о превалировании послеоперационной этиологии заболевания, особенно среди жен- щин молодого, трудоспособного возраста, согласуются с результатами зарубежных исследований [1]. Преобладание женщин в группе послеоперационного гипопаратиреоза (85\%), скорее всего, связано как с большей распространенностью патологии щитовидной железы в данной популяции, так и с большей приверженностью к обследованию и, следовательно, большей частотой проведения хирургических вмешательств на органах шеи. Объем хирургического вмешательства является независимым фактором риска развития хронического гипопаратиреоза, поэтому в структуре предоперационной патологии преобладают ВДРЩЖ и МУЗ [2].

В настоящее время большое внимание уделяется проблеме определения «неконтролируемого» гипопаратиреоза, при этом на первый план выходят не только отклонения в показателях фосфорно-кальциевого обмена, но и наличие ассоциированных осложнений и поиск их предикторов [3].

В проведенном исследовании менее половины пациентов имели целевые показатели кальция и фосфора сыворотки крови (31 и 47\% соответственно). Полученные данные подтверждают отсутствие компенсации заболевания у большинства больных и требуют оптимизации медикаментозного лечения и наблюдения.

При анализе базы данных «НМИЦ эндокринологии» выявлена проблема недостаточного инструментального обследования пациентов - только в 58\% случаев проводился необходимый комплекс диагностических мероприятий. В соответствии с зарубежным консенсусом экспертов по хроническому гипопаратиреозу ежегодное выполнение УЗИ почек, а также динамический осмотр офтальмолога являются необходимым минимумом для своевременной диагностики, профилактики и лечения осложнений $[4,5]$.

Как уже было отмечено в предыдущих исследованиях [6-8], наиболее частым осложнением гипопаратиреоза остается поражение почек. Частота нефрокальциноза и нефролитиаза, по данным различных исследований, варьирует от 12 до 57\%, при этом риски их развития в 5 раз выше общепопуляционных показателей. Сходные результаты отмечены в отношении функции почек. Почечная недостаточность со снижением СКФ менее 60 мл/мин/1,73 м² регистрируется в 12-41\% наблюдений $[6,9]$. В настоящем исследовании установлено, что $13 \%$ пациентов имеют ХБП 3-5 ст., 38\% - поражение структуры почек (нефрокальциноз или нефролитиаз).

Поражение органов зрения с развитием катаракты чаще встречается при нехирургическом гипопаратиреозе (в 4,21 раза, чем в общей популяции), распространенность составляет до 27-55\% [8, 10, 11]. В настоящем исследовании катаракта диагностирована у 14\% больных.

Кальцификация структур головного мозга в популяции пациентов с гипопаратиреозом может наблюдаться более чем в половине случаев (52-74\%) [12]. В настоящем исследовании кальцификация структур головного мозга была выявлена у $10 \%$ обследованных пациентов.

К нередким осложнениям гипопаратиреоза относятся нарушения сердечного ритма [13], которые чаще развиваются вследствие острой гипокальциемии и в основном обратимы при ее купировании. Риски развития психоневрологических нарушений в 2,01-2,45 раза выше, чем в популяции $[8,14]$. По нашим результатам, частота данных осложнений составила $6 \%$ и $10 \%$ соответственно. 
Стандартная терапия гипопаратиреоза включает в себя назначение активных метаболитов/аналогов витамина D в сочетании с препаратами кальция $[4,5]$. По результатам крупных исследований, указанной терапии придерживаются до 88-93\% пациентов с гипопаратиреозом, при этом среднесуточные дозы альфакальцидола составляют 1,0 мкг/сут (0,5; 2,0), кальцитриола - 0,5 мкг/сут (0,125-4,0), препаратов кальция - 1000-2048 мг/сут (800; 9450). Гидрохлоротиазид получают в среднем 19\% больных в средней дозе 31 мг/сут $(12,5 ; 50)$ [8, 15]. В данном исследовании стандартную терапию получали 75\% пациентов. При анализе других схем медикаментозного лечения обращает на себя внимание наличие комбинаций «колекальциферол + препараты кальция», а также «дигидротахистерол + препараты кальция». Все пациенты, получающие данные комбинации, были переведены на терапию активными метаболитами/аналогами витамина D и препаратами кальция.

Нативный витамин D, колекальциферол, использовался в качестве монотерапии гипопаратиреоза в супрафизиологических дозах в период, когда препараты активных метаболитов/аналогов витамина D не были доступны. Недостатком данной схемы лечения является наличие продолжительного периода полувыведения с риском передозировки и интоксикации. Таким образом, назначение нативного витамина D в настоящее время рассматривается при терапии гипопаратиреоза в качестве дополнительного лечения и профилактики сопутствующего дефицита или недостаточности витамина D $[4,5]$.

По современным представлениям, дигидротахистерол не должен применяться для лечения хронического гипопаратиреоза $[4,5]$. При использовании данного препарата значимо возрастает риск гиперкальциемических эпизодов (уровни общего кальция до 3,7-4,97 ммоль/л) затяжного течения в связи с длительным периодом полувыведения данного препарата, зачастую требующих активной инфузионной терапии. К побочным эффектам препарата относят развитие нефрокальциноза и почечной недостаточности $[16,17]$.

Выявленная взаимосвязь между применением больших доз альфакальцидола при отсутствии гиперкальциемии и увеличением суточной кальциурии согласуется с результатами предыдущих исследований [18]. В работе Luis García-Pascual и соавт. при изучении возможных факторов риска развития гиперкальциурии как неблагоприятного прогностического маркера патологии почек при гипопаратиреозе было отмечено, что высокий уровень $1,25(\mathrm{OH})_{2}$ витамина D ассоциирован с более частым выявлением повышенной экскреции кальция с мочой. Повышение уровня 1,25(OH), витамина D свидетельствовало, по-видимому, о передозировке активными метаболитами/аналогами витамина D.

Терапия рекомбинантным человеческим ПТГ (рчПТГ) используется в настоящее время у пациентов с тяжелым, не компенсированным на стандартной терапии гипопаратиреозом. ПтГ (1-34) не зарегистрирован для лечения гипопаратиреоза, однако имеется опыт его применения при этом заболевании в рамках научных исследований. Максимальный период терапии ПтГ (1-34) у детей с нехирургическими формами гипопаратиреоза в настоящее время составляет

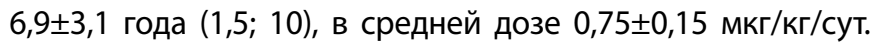
На фоне лечения отмечалось значимое снижение потребности в кальции и препаратах витамина D, положительная динамика маркеров костной резорбции [19]. ПТГ (1-84) на сегодняшний день - единственный препарат рчПтГ, одобренный для лечения гипопаратиреоза в странах Европы и США. В целом период наблюдения за пациентами на терапии ПТГ (1-84) составил около 5 лет, со среднесуточными дозами 50 мкг/сут у большинства пациентов (до 93,9\%). Продемонстрировано, что терапия рчПТГ приводит к нормализации показателей фосфорно-кальциевого обмена при уменьшении потребности в приеме стандартной терапии, вплоть до полной ее отмены [20].

В обследуемой группе 2 пациента получали терапию рчПТГ (1-34). В обоих случаях терапия ПТГ (1-34) была назначена по решению врачебной комиссии пациентам с аутоиммунным гипопаратиреозом в рамках АПС 1 типа в связи с сохранением жизнеугрожающей гипокальциемии, несмотря на применение больших доз стандартной терапии. Сложности в достижении компенсации были вызваны развитием синдрома мальабсорбции. Отмечалась положительная динамика лабораторных показателей и общего самочувствия. На текущий момент в Российской Федерации ПТГ (1-84) не зарегистрирован.

\section{Ограничения исследования}

В данное исследование вошли пациенты с гипопаратиреозом, обратившиеся в ФГБУ «НМИЦ эндокринологии» Минздрава России, таким образом, представленные результаты содержат информацию только по обращаемости в лечебное учреждение.

Результаты кардиологических, офтальмологических и психоневрологических исследований вносились в базу данных на основании консультаций соответствующих специалистов (кардиолога, офтальмолога и психиатра).

\section{ЗАКЛЮЧЕНИЕ}

В настоящее время известно, что пациенты с длительным течением гипопаратиреоза имеют повышенные риски развития осложнений со стороны различных органов и тканей. Анализ представленной базы данных свидетельствует о недостаточной диагностике пациентов с хроническом гипопаратиреозом, особенно в отношении долгосрочных осложнений.

Анализ течения заболевания и структуры выявляемых изменений может помочь в определении прогностических маркеров, в том числе с целью профилактики развития осложнений и формирования критериев компенсации хронического гипопаратиреоза.

\section{ДОПОЛНИТЕЛЬНАЯ ИНФОРМАЦИЯ}

Источник финансирования. Данное исследование выполнено в рамках государственного задания «Всероссийский реестр пациентов с хроническим гипопаратиреозом как основа оптимизации и внедрения персонализированного подхода для улучшения качества оказания медицинской помощи населению Российской Федерации», регистрационный номер НИОКТРАААА-А20-120011790168-2.

Конфликт интересов. Авторы декларируют отсутствие явных и потенциальных конфликтов интересов, связанных с публикацией настоящей статьи.

Участие авторов. Все авторы внесли значимый вклад в проведение исследования и подготовку статьи, прочли и одобрили финальную версию статьи перед публикацией. 


\section{СПИСОК ЛИТЕРАТУРЫ | REFERENCES}

1. Powers J, et al. Prevalence and incidence of hypoparathyroidism in the United States Using a large claims database. J. Bone Miner. Res. 2013;28(12):2570-2576. doi:10.1002/jbmr.2004.

2. Abate EG, Clarke BL. Review of Hypoparathyroidism. Front. Endocrinol. (Lausanne). 2016;7:172. doi:10.3389/fendo.2016.00172.

3. lqbal K, et al. Defining the Characteristics of Chronic Hypoparathyroidism Not Adequately Controlled on Conventional Therapy: Consensus Findings of Three European Delphi Panels. Adv. Ther. 2019;36(11):3007-3016. doi:10.1007/s12325-019-01102-5.

4. Bollerslev J, et al. European Society of Endocrinology Clinical Guideline: Treatment of chronic hypoparathyroidism in adults. Eur. J. Endocrinol. 2015;173(2). doi: 10.1530/EJE-15-0628.

5. Khan AA, et al. Standards of care for hypoparathyroidism in adults: A Canadian and international consensus. Eur. J. Endocrinol. 2019;180(3):1-P22. doi:10.1530/EJE-18-0609.

6. Mitchell DM, et al. Long-term follow-up of patients with hypoparathyroidism. J. Clin. Endocrinol. Metab. 2012;97(12):4507-4514. doi:10.1210/jc.2012-1808.

7. Shoback DM, et al. Presentation of hypoparathyroidism: Etiologies and clinical features. J. Clin. Endocrinol. Metab. 2016;101(6):2300-2312. doi:10.1210/jc.2015-3909.

8. Underbjerg L, et al. Postsurgical hypoparathyroidism-Risk of fractures, Psychiatric Diseases, Cancer, Cataract, and Infections. J. Bone Miner. Res. 2014;29(S1):2504-2510. doi:10.1002/jbmr.2273.

9. Levy l, et al. The impact of hypoparathyroidism treatment on the kidney in children: Long-term retrospective follow-up study. J. Clin. Endocrinol. Metab. 2015;100(11):4106-4113. doi:10.1210/jc.2015-2257.

10. Daba KT, Weldemichael DK, Mulugeta GA. Bilateral hypocalcemic cataract after total thyroidectomy in a young woman: case report. BMC Ophthalmol. 2019;19(1):233. doi:10.1186/s12886-019-1224-9.

11. Saha $S$, et al. Long-term outcome of cataract surgery in patients with idiopathic hypoparathyroidism and its relationship with their calcemic status. J. Bone Miner. Metab. 2017;35(4):405-411. doi:10.1007/s00774-016-0767-6
12. Goswami R, et al. Prevalence and progression of basal ganglia calcification and its pathogenic mechanism in patients with idiopathic hypoparathyroidism. Clin. Endocrinol. (Oxf). 2012;77(2):200-206. doi:10.1111/j.1365-2265.2012.04353.x

13. Newman DB, et al. Reversible cardiac dysfunction associated with hypocalcemia: A systematic review and meta-analysis of individual patient data. Heart Fail. Rev. 2014;19(2):199-205. doi:10.1007/s10741-013-9371-1.

14. Kim SH, et al. Prevalence and complications of nonsurgical hypoparathyroidism in Korea: A nationwide cohort study. PLoS One. 2020;15(5):e0232842. https://doi.org/10.1371/journal.pone.0232842.

15. Underbjerg $L$, et al. Cardiovascular and renal complications to postsurgical hypoparathyroidism: A Danish nationwide controlled historic follow-up study. J. Bone Miner. Res. 2013;28(11):2277-2285. doi:10.1002/jbmr.1979.

16. Jensterle $M$, et al. Dihydrotachysterol intoxication treated with pamidronate: a case report. Cases J. 2010;3(1):78 doi:10.1186/1757-1626-3-78

17. Quack I, et al. Dihydrotachysterol therapy for hypoparathyroidism: consequences of inadequate monitoring. Five cases and a review. Exp. Clin. Endocrinol. diabetes Off.journal, Ger. Soc. Endocrinol. [and] Ger. Diabetes Assoc. Germany, 2005;113(7):376-380. doi:10.1055/s-2005-865724.

18. García-Pascual L, et al. Serum 1,25-Dihydroxyvitamin D as a Biomarker of the Absence of Hypercalciuria in Postsurgical Hypoparathyroidism. J. Clin. Endocrinol. Metab. United States, 2017;102(1):259-266. doi:10.1210/jc.2016-2987

19. Winer K.K, et al. Long-Term Parathyroid Hormone 1-34 Replacement Therapy in Children with Hypoparathyroidism. J. Pediatr. 2018;203):391-399.e1. doi:10.1016/j.jpeds.2018.08.010.

20. Mannstadt M, et al. Safety and Efficacy of 5 Years of Treatment With Recombinant Human Parathyroid Hormone in Adults With Hypoparathyroidism. J. Clin. Endocrinol. Metab. 2019;104(11):5136-5147. doi:10.1210/jc.2019-01010.

Рукопись получена: 24.09.2020. Одобрена к публикации: 25.09.2020. Опубликована online: 24.10.2020.

\section{ИНФОРМАЦИЯ ОБ АВТОРАХ [AUTHORS INFO]}

*Ковалева Елена Владимировна, научный сотрудник отделения патологии околощитовидных желез ФГБУ «НМИЦ эндокринологии» Минздрава России [Elena V. Kovaleva], 117036, г. Москва, ул. Дмитрия Ульянова, д. 11, http://orcid.org/0000-0002-9258-2591, SPIN-код: 7387-6791, e-mail: elen.v.kovaleva@gmail.com

Айнетдинова Алина Ринатовна, врач-статистик отделения патологии околощитовидных желез ФГБУ «НМИЦ эндокринологии» Минздрава России [Alina R. Ajnetdinova]; адрес: Россия, 117036, Москва, ул. Дм. Ульянова, д. 11 [address: 11 Dm. Ulyanova street, 117036 Moscow, Russia], ORCID: 0000-0001-6935-3187, eLibrary SPIN: 9617-7460, e-mail: 9803005@mail.ru

Еремкина Анна Константиновна, к.м.н., старший научный сотрудник, и.о. заведующей отделением патологии околощитовидных желез ФГБУ «НМИЦ эндокринологии» Минздрава России [Anna K. Eremkina]; адрес: Россия, 117036, Москва, ул. Дм. Ульянова, д. 11 [address: 11 Dm. Ulyanova street, 117036 Moscow, Russia]; ORCID: https://orcid.org/0000-0001-6667-062X; eLibrary SPIN: 8848-2660; e-mail: a.lipatenkova@gmail.com Мокрышева Наталья Георгиевна, д.м.Н., член-корр., профессор РАН, директор ФГБУ «НМИЦ эндокринологии» Минздрава России [Natalia G. Mokrysheva, MD, PhD]; ORCID: http://orcid.org/0000-0002-9717-9742; eLibrary SPIN: 5624-3875; e-mail: nm70@mail.ru

\section{ЦИТИРОВАТЬ:}

Ковалева Е.В., Еремкина А.К., Айнетдинова А.Р., Мокрышева Н.Г. Результаты анализа базы данных пациентов с гипопаратиреозом ФГБУ «НМИЦ эндокринологии» Минздрава России // Проблемы эндокринологии. - 2020. T. 66. — №5. — C. 7-14. doi: https://doi.org/10.14341/probl12675

\section{TO CITE THIS ARTICLE:}

Kovaleva EV., Ajnetdinova AR., Eremkina AK., Mokrysheva NG. Evaluation of chronic hypoparathyroidism course according to the Database of Endocrinology Research Centre. Problems of Endocrinology. 2020;66(5):7-14. doi: https://doi.org/10.14341/probl12675 